\title{
Do regular attenders have better oral health?
}

\section{The impact of attendance patterns on oral health in a general dental practice}

\section{W. Richards and J. Ameen Br Dent J 2002; 193: 697-702}

\section{Objective}

The aim of this study was to examine the impact of attendance patterns on oral health in the context of government policy on dental care and registration in the UK.

\section{Method}

The data involved 643 consecutive patient responses to a questionnaire on dental health taken from a survey that was conducted during 1998 in an urban area of Swansea. The survey continued for a period of six months and covered patients of 18 years of age and over, responding to a questionnaire on the subjective oral health status indicators including the pattern of their attendance to dental practices.

\section{Results}

Regular dental care attendance has a significant positive impact on dental health while its impact on the number of teeth present is insignificant. Regular attendees also suffer significantly less from the severity, prevalence, social and psychological impacts of dental health problems.

Conclusion

Regular dental attendance is associated with better oral health when regularity of care is defined as a visit within a two-year period. The rationality of a 15 month registration period is therefore debatable in the context of developing equitable services.

\section{IN BRIEF}

- Regular care is defined as a visit for care within two years.

- Oral health is defined within the psychosocial framework of 'discomfort, disabiliy and discontent'.

- Significantly more registered patients are regular than irregular attenders.

- Significantly more regular attendees are healthier compared with irregular and new patients.

- A 15 month registration time frame is inconsistent with developing equitable services.

\section{COMMENT}

This paper describes a study which uses an empirical measure of oral well being which reflects contemporary definitions of health. The data are analysed in order to examine the effect of regular dental attendance in a population of dental practice patients. Data from a total of 638 usable questionnaires indicated that a sizeable minority of the patients attending the dental practice had problems with their mouths and teeth which impacted on their lives. Almost half of the respondents had suffered pain and two thirds had experienced other symptoms in the month before being questioned. Paradoxically, two thirds of the respondents also thought that their oral health was good or excellent.

Those who are unfamiliar with the SOHSI measurement tool used in this study will perhaps need to familiarise themselves with its content in order that the importance of Professor Richards' results can be fully understood. Of course, cross-sectional studies must be carefully interpreted, particularly when examining the effects of dental attendance, as non-attenders will be under represented in the sample and even the 'non-regular' attenders who are included, are not representative of the people who never attend, or only attend in the direst circumstances. Thus, lower social class patients are also under represented. The known relationship between oral health and social class, therefore, acts as a confounder and it is difficult to separate the effects of the two variables: social class and attendance.

Despite this problem, which is recognised by the authors, this genuinely general practice based study offers an extremely helpful contribution to the 'frequency of attendance' debate. It uses objectively measured attendance rather than the more commonly used 'reported' attendance. Furthermore, the paper is significant in that it uses a patient centred measure of oral well being as the focus of the study, rather than, as has traditionally been the case, using normatively measured disease as the outcome variable. This reflects a shift in thinking which occurred some time ago in medicine and is now beginning to happen in dentistry. This paper recognises that health is a subjective and experiential entity. It shows that dentists and healthcare personnel enhance people's well being, rather than simply 'mending' body parts which have broken down. It is to be hoped that scientific studies in practices will follow the lead that has been given by this paper.

Elizabeth J Kay, Professor of Dental Health Services Research, University of Manchester 